\title{
Perspectives on Rehabilitation Using Non-invasive Brain Stimulation Based on Second-Person Neuroscience of Teaching-Learning Interactions
}

\section{OPEN ACCESS}

Edited by: Yafeng Pan,

Karolinska Institutet (KI), Sweden

Reviewed by: Lifen Zheng,

Beijing Normal University, China

Takahiko Koike,

National Institute for Physiological Sciences (NIPS), Japan

*Correspondence: Naoyuki Takeuchi n-take@hs.akita-u.ac.jp

Specialty section: This article was submitted to Neuropsychology, a section of the journal Frontiers in Psychology

Received: 05 October 2021 Accepted: 24 November 2021

Published: 05 January 2022

Citation:

Takeuchi N (2022) Perspectives on Rehabilitation Using Non-invasive Brain Stimulation Based on SecondPerson Neuroscience of TeachingLearning Interactions.

Front. Psychol. 12:789637. doi: 10.3389/fpsyg.2021.789637

\author{
Naoyuki Takeuchi* \\ Department of Physical Therapy, Akita University Graduate School of Health Sciences, Akita, Japan
}

Recent advances in second-person neuroscience have allowed the underlying neural mechanisms involved in teaching-learning interactions to be better understood. Teaching is not merely a one-way transfer of information from teacher to student; it is a complex interaction that requires metacognitive and mentalizing skills to understand others' intentions and integrate information regarding oneself and others. Physiotherapy involving therapists instructing patients on how to improve their motor skills is a clinical field in which teaching-learning interactions play a central role. Accumulating evidence suggests that non-invasive brain stimulation (NIBS) modulates cognitive functions; however, NIBS approaches to teaching-learning interactions are yet to be utilized in rehabilitation. In this review, I evaluate the present research into NIBS and its role in enhancing metacognitive and mentalizing abilities; I then review hyperscanning studies of teaching-learning interactions and explore the potential clinical applications of NIBS in rehabilitation. Dual-brain stimulation using NIBS has been developed based on findings of brain-to-brain synchrony in hyperscanning studies, and it is delivered simultaneously to two individuals to increase inter-brain synchronized oscillations at the stimulated frequency. Artificial induction of brain-to-brain synchrony has the potential to promote instruction-based learning. The brain-to-brain interface, which induces inter-brain synchronization by adjusting the patient's brain activity, using NIBS, to the therapist's brain activity, could have a positive effect on both therapist-patient interactions and rehabilitation outcomes. NIBS based on second-person neuroscience has the potential to serve as a useful addition to the current neuroscientific methods used in complementary interventions for rehabilitation.

\footnotetext{
Keywords: non-invasive brain stimulation, second-person neuroscience, instruction-based learning, metacognitive function, mentalizing, rehabilitation
}

\section{INTRODUCTION}

Teaching plays a central role in physiotherapy as physiotherapists instruct patients on how to improve their motor skills. Teaching-learning interactions are not a simple one-way transfer of information, but rather a complex process that involves speculation, evaluation, and mutual understanding between teachers and students (Rodriguez, 2013; Liu et al., 2019a). Speculating 
about the student's behavior and learning state is a critical aspect of teaching and is related to the ability to understand the intentions of others, known as mentalizing (Strauss et al., 2014; Kline, 2015). In addition, teachers need metacognitive skills to monitor and regulate their own cognitive processes (Kline, 2015; Takeuchi et al., 2017). Similarly, students are not mere listeners or receivers of knowledge from their teachers. Students need metacognitive skills in order to learn, to monitor what they know, and understand what they are capable of doing (Chatzipanteli et al., 2015; Takeuchi et al., 2019). Students also need to consider the teacher's intentions, even if they are not required to think deeply about the teacher's mental state (Strauss et al., 2014; Zheng et al., 2018).

Many brain regions, including the prefrontal cortex (PFC), inferior frontal gyrus (IFG), temporo-parietal junction (TPJ), and medial precuneus are involved in metacognitive and mentalizing processes (Vogeley, 2017; Vaccaro and Fleming, 2018; Arioli and Canessa, 2019). These brain regions have been investigated as potential targets to modulate metacognitive and mentalizing skills via non-invasive brain stimulation (NIBS) techniques that can alter cortical excitability, such as repetitive transcranial magnetic stimulation (rTMS) and transcranial direct current stimulation (tDCS; Sowden et al., 2015; Chua et al., 2017; Gaynor and Chua, 2019; Martin et al., 2021). Recently, transcranial alternating current stimulation (tACS), which can non-invasively modulate oscillatory brain activity, has attracted attention as a promising technique for modulating social functions (Bramson et al., 2020). Metacognitive and mentalizing functions are impaired after brain damage, such as stroke and traumatic brain injury, and patients with brain damage often need rehabilitation (Martín-Rodríguez and León-Carrión, 2010; Al Banna et al., 2016; Nijsse et al., 2019; Yeo et al., 2021). Moreover, these social impairments are associated with poor rehabilitation outcomes. Therefore, NIBS approaches aimed at modulating metacognitive and mentalizing functions may enhance rehabilitation outcomes by facilitating therapist-patient interactions. However, to date, NIBS approaches used in rehabilitation have primarily focused on ameliorating patients' motor and cognitive deficits (Takeuchi and Izumi, 2012; Ahorsu et al., 2021; Madrid and Benninger, 2021), and methods targeting interpersonal interactions in the teaching-learning process remain unexplored.

This review explores the potential for applying NIBS techniques in rehabilitation settings using second-person neuroscience approaches in teaching-learning interactions. First, I review studies using NIBS on single brains to enhance patients' metacognitive, mentalizing, and imitation abilities. Next, I review brain-to-brain synchrony during teaching-learning interactions revealed by hyperscanning, which is a neuroimaging technique used to measure the activity of multiple brains simultaneously. Then, I discuss the potential for dual-brain stimulation using tACS to modulate interpersonal interactions related to instruction-

Abbreviations: IFG, inferior frontal gyrus; NIBS, non-invasive brain stimulation; IPL, inferior parietal lobule; PAC, phase-amplitude coupling; PFC, prefrontal cortex; rTMS, repetitive transcranial magnetic stimulation; tACS, transcranial alternating current stimulation; tDCS, transcranial direct current stimulation; TPJ, temporo-parietal junction. based learning. Hyper-tACS in two individuals has been developed to induce inter-brain synchronized oscillation frequencies. The concurrent manipulation of brain activities helps elucidate the causal role of brain-to-brain synchrony in the teaching-learning process. Moreover, artificial induction of brain-to-brain synchrony may induce pro-social effects and enhance instruction-based learning. However, the use dual-brain stimulation to operate the therapist's brain in real-word rehabilitation settings is unrealistic. Finally, to inform future physiotherapy research, considering therapist-patient interactions, I discuss the potential for using brain-to-brain interfaces, which allow two brains to mutually exchange decoded neural information, and the feedback of brainto-brain synchrony to produce better rehabilitation outcomes.

\section{SINGLE-BRAIN APPROACHES USING NIBS}

Convergent evidence from a number of neuroimaging studies has suggested that the PFC plays a critical role in metacognitive processes (Fleming and Dolan, 2012; Vaccaro and Fleming, 2018). Hyperscanning studies of the teaching-learning process have shown that the PFC plays a central role in metacognitive function in both teachers and students (Takeuchi et al., 2017, 2019). Several studies have shown that NIBS over the PFC facilitates metacognitive processes, such as assessments of own knowledge. Chua et al. reported that excitatory anodal tDCS of the dorsolateral PFC improved the accuracy of participants' general knowledge metamemory more than sham or active stimulation of the anterior temporal lobe (Chua et al., 2017). Gaynor et al. also reported that anodal tDCS of the anterior PFC improved the accuracy of participants' judgments of learning in encoding low-frequency and inverted words, compared with sham or active stimulation of the dorsolateral PFC. However, anodal tDCS of the anterior PFC decreased participants' judgments of learning accuracy in encoding high-frequency words relative to sham stimulation; these findings suggest that the role of the anterior PFC in the subjective level of confidence in metamemory varies according to task difficulty (Gaynor and Chua, 2019). Ryals et al. reported that bilateral continuous theta burst stimulation of the anterior PFC improved judgments of learning accuracy in an associative recognition task more than bilateral dorsolateral PFC or vertex stimulation (Ryals et al., 2016). Thus, NIBS of the PFC may be a potential adjuvant approach for facilitating instruction-based learning by enhancing metacognitive function; however, it should be noted that the effect of NIBS on the PFC varies greatly depending on the task and stimulation method.

The mentalizing system, which allows people to understand the intentions of others by making inferences about their thoughts and beliefs, has been reported to engage a range of brain regions, including the TPJ (especially the right hemisphere), the medial PFC (mPFC), and the medial precuneus (Vogeley, 2017; Arioli and Canessa, 2019). Sowden et al. showed that tDCS of the right TPJ (rTPJ) enhanced mentalizing performance. Excitatory anodal tDCS of the rTPJ improved participants' accuracy at detecting whether a person was lying, specifically when the 
person's own views conflicted with others' views (Sowden et al., 2015). The mPFC is also a potential target that could be triggered to facilitate the mentalizing process using NIBS. Manfredi et al. reported that anodal tDCS of the mPFC improved participants' abilities to recognize humorous situations that required mentalizing skills in order to be understood (Manfredi et al., 2020). Martin et al. investigated whether $\mathrm{tDCS}$ of the MPFC enhances mentalizing functions using the cross-cultural version of the Reading the Mind in the Eyes test. Anodal tDCS of the mPFC enhanced mentalizing performance, and this improvement was greater in participants with less cross-cultural contact (Martin et al., 2021).

In addition to the mentalizing system, the mirror neuron system that maps observed actions into the observer's own motor representations is thought to be involved in the neural mechanism underlying understanding others' intentions (Rizzolatti et al., 2014; Cole and Barraclough, 2018). Several brain imaging studies have reported that the IFG, premotor cortex, inferior parietal lobule (IPL), and superior parietal lobule are the main nodes of the mirror neuron system (Caspers et al., 2010; Molenberghs et al., 2012; Vogeley, 2017). Few studies have used NIBS to investigate the role of the mirror neuron system in the mentalizing process compared with the roles of the rTPJ and mPFC (Yao et al., 2021). However, several studies have reported that NIBS of the mirror neuron system could modulate imitation performance, which may be a promising technique to help patients acquire new motor skills during physiotherapy. Weiss et al. reported that anodal tDCS of the left IPL improved the matching task of hand gestures (Weiss et al., 2013). Vanbellingen et al. investigated whether dual-site stimulation, which consists of left IFG stimulation immediately followed by right IPL stimulation, modulated the imitation process. The results indicated that dual-site stimulation using continuous theta bursts enhanced the performance of gesture imitation compared with sham or left IFG stimulation (Vanbellingen et al., 2020). Consequently, the tACS approach to brain communication has attracted attention for modulating the imitation process. It has been reported that tACS of distant cortical regions can effectively modulate oscillatory phase synchrony and functional connectivity between the targeted brain regions via entrainment of brain oscillations (CabralCalderin and Wilke, 2020). Takeuchi et al. investigated whether the manipulation of brain communication in the frontoparietal mirror network altered imitation performance by applying gamma and theta phase-amplitude coupling (PAC) tACS to both the left IFG and left IPL. The application of in-phase theta-gamma PAC tACS over the left frontoparietal mirror network significantly improved participants' gesture matching and meaningless gesture imitation performance relative to their baseline performance (Takeuchi et al., 2021). Theta-gamma PAC brain oscillations are hypothesized to coordinate activity in distant brain regions and induce effective communication during cognitive processing in humans (Canolty et al., 2006). Therefore, the increased synchrony between components of the left frontoparietal mirror network induced by in-phase theta-gamma PAC tACS may facilitate the imitation process by strengthening network efficiency. In addition to the mirror neuron system, it is thought that brain communication between distant regions, such as the mPFC and rTPJ, works together for the mentalizing process (Hill et al., 2017). It is worth considering whether dual-site stimulation to these distant brain regions enhances mentalizing ability more than single-site stimulation.

The results of NIBS studies suggest that manipulation of brain activities and communication related to metacognition, mentalizing, and imitation functions may be a potential approach to enhance the teaching-learning process in physiotherapy. However, the target of the NIBS approaches discussed in this chapter is mainly the single brain of a patient whose cognitive functions are impaired, although NIBS may also indirectly affect therapist-patient interactions. In the next chapter, I will focus on the NIBS approach used to target two brains in the context of therapist-patient interactions.

\section{TWO-BRAIN APPROACHES USING NIBS}

In the teaching-learning process, facial expressions and hand gestures, which are coupled to the teacher's speech signals, may enhance the coupling of the student's sensory system to the teacher's motor system (Hasson et al., 2012; Hoehl et al., 2021). Social behaviors, such as mutual eye contact and joint attention during the learning process, also likely play a role in synchronizing neural responses in teachers and students (Dikker et al., 2014; Leong et al., 2017). Hyperscanning studies have demonstrated brain-to-brain synchrony during teaching-learning interactions (Zheng et al., 2018; Liu et al., 2019a; Pan et al., 2020). Using functional near-infrared spectroscopy, Liu et al. reported that the brain activities in the left PFC synchronized in teachers and students when they participated in an instruction-based learning task provided that students had prior knowledge. Moreover, brain-to-brain synchrony early in the teaching process is correlated with improved teaching effectiveness (Liu et al., 2019a). Pan et al. showed that brain-to-brain synchrony in the PFC and the superior temporal cortex during instruction-based learning tasks was correlated with better learning outcomes (Pan et al., 2020). Oscillatory synchronization through phase alignment may facilitate more efficient information transfer between brains, resulting in better teaching-learning interactions (Lakatos et al., 2019; Wass et al., 2020). Moreover, brain-to-brain synchrony may either cause or be caused by behavioral entrainment, cognitive alignment, and affective contagion during teaching-learning interactions, and these factors may work jointly to promote learning performance (Kingsbury and Hong, 2020; Pan et al., 2021a).

Artificial induction of brain-to-brain synchrony has the potential to promote instruction-based learning. It is well known that motor and sensory synchronization can cause brain-to-brain synchrony (Rennung and Göritz, 2016). Physical movement synchrony between teachers and students during instructionbased learning tasks has been associated with a higher perception of rapport and better learning performance in students (Zhou, 2012). However, brain-to-brain synchrony may be a byproduct of the behavioral and perceptual similarities between individuals during learning. Moreover, it should be noted that findings of brain-to-brain synchrony do not indicate a direct connection between brains. Therefore, to investigate the causal role of 
brain-to-brain synchrony in the teaching-learning process, it is essential to simultaneously manipulate brain activity in interacting partners and observe the subsequent effects on learning. At present, tACS is the main type of NIBS utilized for dual-brain stimulation to induce brain-to-brain synchrony in human studies (Novembre and Iannetti, 2021) because tACS has a feature that can modulate oscillatory phase synchrony and functional connectivity between targeted brain regions by entraining brain oscillations (Vosskuhl et al., 2018; Cabral-Calderin and Wilke, 2020). As an extension of the intra-brain to inter-brain communication approach, tACS applied simultaneously to two individuals (hyper-tACS) has been developed to modulate interbrain synchronized oscillation frequency and artificially operate interpersonal interactions. Pan et al. investigated whether hypertACS of the IFG facilitated the teaching-learning process. They found that in-phase theta hyper-tACS in both the instructor and the learner augmented social interactive learning during a naturalistic song-learning task (Pan et al., 2021b). Moreover, hyper-tACS synchronized body movements between the instructor and the learner. Interpersonal movement synchrony is known to induce pro-social effects, such as rapport, cooperation, and affiliation (Keller et al., 2014; Reddish et al., 2016). These social factors might have a positive impact on the teaching-learning process and rehabilitation outcomes (Hall et al., 2010; Zee et al., 2013; Kinney et al., 2020). Considering these findings, hypertACS may enhance instruction-based learning in physiotherapy. In addition, the concurrent manipulation of brain activities using dual-brain stimulation methods, such as hyper-tACS, may help elucidate the causal role of therapist-patient brain-to-brain synchrony in the teaching-learning process, which cannot be achieved by hyperscanning and single-brain stimulation studies (Novembre and Iannetti, 2021).

However, there are several problems associated with hypertACS. First, tACS has been shown to be strongly affected by scalp sensory stimulation (Asamoah et al., 2019). Therefore, brain regions unrelated to cognitive functions must also be investigated as control sites in order to rule out the possibility that the observed positive effect of hyper-tACS on interpersonal interactions is merely derived from peripheral stimulation. Second, some doubt remains as to whether hyper-tACS can facilitate interpersonal interactions. Szymanski et al. showed that in-phase theta hypertACS of the left frontocentral and centroparietal sites during joint action impaired rather than improved performance during a dyadic drumming synchrony task (Szymanski et al., 2017). Although it is possible that the frequency and site of tACS were inappropriate for the dyadic task, this negative result suggests that the frequency entrained by tACS may differ between individuals, resulting in a reduction in dyadic synchrony. Third, participants' brain activity during the teaching-learning process may not be completely synchronized in time and space. Zheng et al. reported that better learning performance was associated with stronger brain-to-brain synchrony between the rTPJ of the teacher and the anterior superior temporal cortex of the student when the teacher's brain activity preceded that of the student (Zheng et al., 2018). Future studies aimed at promoting interpersonal interactions using hyper-tACS must take into account these temporal and spatial asymmetries in brain-to-brain synchrony.
Hyper-tACS may induce pro-social effects and enhance instruction-based learning via brain-to-brain synchrony. However, using NIBS to operate the therapist's brain is not realistic in a real-world rehabilitation setting considering the ethical issues regarding manipulating healthy individuals' brains. To further address the issues associated with hyper-tACS, I discuss in the final chapter of this paper the implications of using brain-tobrain interfaces, which allow the mutual exchange of decoded neural information between two brains, and the feedback of brain-to-brain synchrony to produce better rehabilitation outcomes.

\section{THE FUTURE OF PHYSIOTHERAPY USING TWO-PERSON NEUROSCIENCE APPROACHES IN THERAPIST-PATIENT INTERACTIONS}

A new neural interface technology, i.e., the brain-to-brain interface, could induce brain-to-brain synchrony by operating the patient's brain activity using NIBS and adjusting it to the therapist's brain activity. A brain-to-brain interface allows two brains to mutually exchange decoded neural information with each other using a brain-computer interface that reads the sender's brain activity and a computer-brain interface that transmits the neural information to the receiver's brain using electrical stimulation (Nam et al., 2021). The direct transmission of information from a therapist's to a patient's brain via a brain-to-brain interface may facilitate instruction-based learning and more complex bidirectional therapist-patient interactions. In addition to brain stimulation, cross-brain neurofeedback is also a promising approach for manipulating brain-to-brain synchrony. A recent study showed that audiovisual feedback of brain-to-brain synchrony leads to increased inter-brain coupling (Dikker et al., 2021). Moreover, the study indicated that brainto-brain synchrony was positively associated with social behaviors, such as eye contact and joint action. Considering these findings, NIBS assist and/or feedback of brain-to-brain synchrony may be a novel and useful rehabilitation technique to support patients' learning by enhancing pro-social behavior. Feedback from brainto-brain synchrony may also aid less experienced therapists (e.g., therapy students and novice therapists) in predicting the learning success of their patients. However, it is not clear whether the brains of therapists and patients synchronize during instruction-based learning in the same way that the brains of healthy subjects do. Future studies are required to determine whether brain-to-brain synchrony of therapists and patients alters according to patients' learning performance.

Lastly, a cautionary point regarding therapeutic relationships must be considered before NIBS based on second-person neuroscience is used in physiotherapy. It is well known that the state of the therapist-patient relationship influences the effectiveness of psychotherapy (Koole and Tschacher, 2016). In physiotherapy, better therapeutic relationships are associated with improved rehabilitation outcomes (Hall et al., 2010; Kinney et al., 2020), and the therapeutic relationship between physiotherapists and patients is considered to be important in 
the field of physiotherapy (Søndenå et al., 2020; Bishop et al., 2021). Artificial induction of brain-to-brain synchrony using NIBS is expected to improve social interpersonal relationships in dyads of lovers (Liu et al., 2019b). However, the application of NIBS technologies to clinical fields to modulate the social interactions between therapists and patients raises important ethical issues and highlights the need for neuroethics guidelines (Cohen Kadosh et al., 2012; Ienca et al., 2017). Before NIBS approaches are used to improve the alliance between therapists and patients, it is necessary to first discuss the problems surrounding artificial manipulation of the therapist-patient relationship and ensure that the therapist's neutrality toward the patient is maintained.

In conclusion, despite the translational potential of NIBS based on second-person neuroscience approaches to the rehabilitation field, the methodology remains in its infancy and poses several issues that will require greater innovation,

\section{REFERENCES}

Ahorsu, D. K., Adjaottor, E. S., and Lam, B. Y. H. (2021). Intervention effect of non-invasive brain stimulation on cognitive functions among people with traumatic brain injury: A systematic review and meta-analysis. Brain Sci. 11:840. doi: 10.3390/brainsci11070840

Al Banna, M., Redha, N. A., Abdulla, F., Nair, B., and Donnellan, C. (2016). Metacognitive function poststroke: a review of definition and assessment. J. Neurol. Neurosurg. Psychiatry 87, 161-166. doi: 10.1136/jnnp-2015-310305

Arioli, M., and Canessa, N. (2019). Neural processing of social interaction: coordinate-based meta-analytic evidence from human neuroimaging studies. Hum. Brain Mapp. 40, 3712-3737. doi: 10.1002/hbm.24627

Asamoah, B., Khatoun, A., and Mc Laughlin, M. (2019). tACS motor system effects can be caused by transcutaneous stimulation of peripheral nerves. Nat. Commun. 10:266. doi: 10.1038/s41467-018-08183-w

Bishop, M., Kayes, N., and Mcpherson, K. (2021). Understanding the therapeutic alliance in stroke rehabilitation. Disabil. Rehabil. 43, 1074-1083. doi: 10.1080/09638288.2019.1651909

Bramson, B., Den Ouden, H. E., Toni, I., and Roelofs, K. (2020). Improving emotional-action control by targeting long-range phase-amplitude neuronal coupling. elife 9:e59600. doi: 10.7554/eLife.59600

Cabral-Calderin, Y., and Wilke, M. (2020). Probing the link between perception and oscillations: lessons from transcranial alternating current stimulation. Neuroscientist 26, 57-73. doi: 10.1177/1073858419828646

Canolty, R. T., Edwards, E., Dalal, S. S., Soltani, M., Nagarajan, S. S., Kirsch, H. E., et al. (2006). High gamma power is phase-locked to theta oscillations in human neocortex. Science 313, 1626-1628. doi: 10.1126/science. 1128115

Caspers, S., Zilles, K., Laird, A. R., and Eickhoff, S. B. (2010). ALE metaanalysis of action observation and imitation in the human brain. NeuroImage 50, 1148-1167. doi: 10.1016/j.neuroimage.2009.12.112

Chatzipanteli, A., Digelidis, N., Karatzoglidis, C., and Dean, R. (2015). Promoting students' metacognitive behavior in physical education through TGFU. Am. J. Educ. Res. 1, 28-36.

Chua, E. F., Ahmed, R., and Garcia, S. M. (2017). Effects of HD-tDCS on memory and metamemory for general knowledge questions that vary by difficulty. Brain Stimul. 10, 231-241. doi: 10.1016/j.brs.2016.10.013

Cohen Kadosh, R., Levy, N., O'shea, J., Shea, N., and Savulescu, J. (2012). The neuroethics of non-invasive brain stimulation. Curr. Biol. 22, R108-R111. doi: $10.1016 /$ j.cub.2012.01.013

Cole, E. J., and Barraclough, N. E. (2018). Timing of mirror system activation when inferring the intentions of others. Brain Res. 1700, 109-117. doi: 10.1016/j.brainres.2018.07.015

Dikker, S., Michalareas, G., Oostrik, M., Serafimaki, A., Kahraman, H. M., Struiksma, M. E., et al. (2021). Crowdsourcing neuroscience: inter-brain both methodologically and ethically, before NIBS can be adopted into physiotherapy practices. This review identified the topics that will be instrumental in advancing neuroscientific knowledge of the instruction-based learning that is essential to physiotherapy.

\section{AUTHOR CONTRIBUTIONS}

The author confirms being the sole contributor of this work and has approved it for publication.

\section{FUNDING}

This work was supported by a Grant-in-Aid for Scientific Research (no. 20K11278) from the Japan Society for the Promotion of Science.

coupling during face-to-face interactions outside the laboratory. NeuroImage 227:117436. doi: 10.1016/j.neuroimage.2020.117436

Dikker, S., Silbert, L. J., Hasson, U., and Zevin, J. D. (2014). On the same wavelength: predictable language enhances speaker-listener brain-to-brain synchrony in posterior superior temporal gyrus. J. Neurosci. 34, 6267-6272. doi: 10.1523/JNEUROSCI.3796-13.2014

Fleming, S. M., and Dolan, R. J. (2012). The neural basis of metacognitive ability. Philos. Trans. R. Soc. Lond. B Biol. Sci. 367, 1338-1349. doi: 10.1098/rstb.2011.0417

Gaynor, A. M., and Chua, E. F. (2019). Transcranial direct current stimulation over the prefrontal cortex alters encoding and judgments of learning based on fluency. J. Cogn. Neurosci. 31, 1710-1725. doi: 10.1162/jocn_a_01449

Hall, A. M., Ferreira, P. H., Maher, C. G., Latimer, J., and Ferreira, M. L. (2010). The influence of the therapist-patient relationship on treatment outcome in physical rehabilitation: a systematic review. Phys. Ther. 90, 1099-1110. doi: $10.2522 /$ ptj.20090245

Hasson, U., Ghazanfar, A. A., Galantucci, B., Garrod, S., and Keysers, C. (2012). Brain-to-brain coupling: a mechanism for creating and sharing a social world. Trends Cogn. Sci. 16, 114-121. doi: 10.1016/j.tics.2011.12.007

Hill, C. A., Suzuki, S., Polania, R., Moisa, M., O'doherty, J. P., and Ruff, C. C. (2017). A causal account of the brain network computations underlying strategic social behavior. Nat. Neurosci. 20, 1142-1149. doi: 10.1038/nn.4602

Hoehl, S., Fairhurst, M., and Schirmer, A. (2021). Interactional synchrony: signals, mechanisms and benefits. Soc. Cogn. Affect. Neurosci. 16, 5-18. doi: $10.1093 /$ scan/nsaa024

Ienca, M., Kressig, R. W., Jotterand, F., and Elger, B. (2017). Proactive ethical design for neuroengineering, assistive and rehabilitation technologies: the Cybathlon lesson. J. Neuroeng. Rehabil. 14:115. doi: 10.1186/s12984-017-0325-z

Keller, P. E., Novembre, G., and Hove, M. J. (2014). Rhythm in joint action: psychological and neurophysiological mechanisms for real-time interpersonal coordination. Philos. Trans. R. Soc. Lond. Ser. B Biol. Sci. 369:20130394. doi: $10.1098 / \mathrm{rstb} .2013 .0394$

Kingsbury, L., and Hong, W. (2020). A multi-brain framework for social interaction. Trends Neurosci. 43, 651-666. doi: 10.1016/j.tins.2020.06.008

Kinney, M., Seider, J., Beaty, A. F., Coughlin, K., Dyal, M., and Clewley, D. (2020). The impact of therapeutic alliance in physical therapy for chronic musculoskeletal pain: A systematic review of the literature. Physiother. Theory Pract. 36, 886-898. doi: 10.1080/09593985.2018.1516015

Kline, M. A. (2015). How to learn about teaching: An evolutionary framework for the study of teaching behavior in humans and other animals. Behav. Brain Sci. 38:e31. doi: 10.1017/S0140525X14000090

Koole, S. L., and Tschacher, W. (2016). Synchrony in psychotherapy: A review and an integrative framework for the therapeutic alliance. Front. Psychol. 7:862. doi: $10.3389 /$ fpsyg.2016.00862

Lakatos, P., Gross, J., and Thut, G. (2019). A new unifying account of the roles of neuronal entrainment. Curr. Biol. 29, R890-R905. doi: 10.1016/j. cub.2019.07.075 
Leong, V., Byrne, E., Clackson, K., Georgieva, S., Lam, S., and Wass, S. (2017). Speaker gaze increases information coupling between infant and adult brains. Proc. Natl. Acad. Sci. U. S. A. 114, 13290-13295. doi: 10.1073/pnas.1702493114

Liu, S., Ma, R., Liu, X., Zhang, C., Chen, Y., Jin, C., et al. (2019b). Using transcranial alternating current stimulation (tACS) to improve romantic relationships can be a promising approach. Front. Psychol. 10:365. doi: 10.3389/fpsyg.2019.00365

Liu, J., Zhang, R., Geng, B., Zhang, T., Yuan, D., Otani, S., et al. (2019a). Interplay between prior knowledge and communication mode on teaching effectiveness: interpersonal neural synchronization as a neural marker. NeuroImage 193, 93-102. doi: 10.1016/j.neuroimage.2019.03.004

Madrid, J., and Benninger, D. H. (2021). Non-invasive brain stimulation for Parkinson's disease: clinical evidence, latest concepts and future goals: A systematic review. J. Neurosci. Methods 347:108957. doi: 10.1016/j.jneumeth.2020.108957

Manfredi, M., Proverbio, A. M., Marques, L. M., Ribeiro, B., Morello, Y. N., and L., and Boggio, P.S., (2020). Anodal transcranial direct current stimulation of MPFC enhances humor processing. Soc. Neurosci. 15, 199-213. doi: 10.1080/17470919.2019.1674687

Martin, A. K., Su, P., and Meinzer, M. (2021). Improving cross-cultural "mindreading" with electrical brain stimulation. Neuroscience 455, 107-112. doi: 10.1016/j.neuroscience.2020.12.007

Martín-Rodríguez, J. F., and León-Carrión, J. (2010). Theory of mind deficits in patients with acquired brain injury: a quantitative review. Neuropsychologia 48, 1181-1191. doi: 10.1016/j.neuropsychologia.2010.02.009

Molenberghs, P., Cunnington, R., and Mattingley, J. B. (2012). Brain regions with mirror properties: a meta-analysis of 125 human fMRI studies. Neurosci. Biobehav. Rev. 36, 341-349. doi: 10.1016/j.neubiorev.2011.07.004

Nam, C. S., Traylor, Z., Chen, M., Jiang, X., Feng, W., and Chhatbar, P. Y. (2021). Direct communication between brains: A systematic PRISMA review of brainto-brain interface. Front. Neurorobot. 15:656943. doi: 10.3389/fnbot.2021.656943

Nijsse, B., Spikman, J. M., Visser-Meily, J. M. A., De Kort, P. L. M., and Van Heugten, C. M. (2019). Social cognition impairments are associated with behavioural changes in the long term after stroke. PLoS One 14:e0213725. doi: 10.1371/journal.pone.0213725

Novembre, G., and Iannetti, G. D. (2021). Hyperscanning alone cannot prove causality. Multibrain stimulation can. Trends Cogn. Sci. 25, 96-99. doi: 10.1016/j.tics.2020.11.003

Pan, Y., Dikker, S., Goldstein, P., Zhu, Y., Yang, C., and Hu, Y. (2020). Instructorlearner brain coupling discriminates between instructional approaches and predicts learning. NeuroImage 211:116657. doi: 10.1016/j.neuroimage.2020.116657

Pan, Y., Novembre, G., and Olsson, A. (2021a). The interpersonal neuroscience of social learning. PsyArXiv. doi: 10.31234/osf.io/p386k

Pan, Y., Novembre, G., Song, B., Zhu, Y., and Hu, Y. (2021b). Dual brain stimulation enhances interpersonal learning through spontaneous movement synchrony. Soc. Cogn. Affect. Neurosci. 16, 210-221. doi: 10.1093/scan/nsaa080

Reddish, P., Tong, E. M., Jong, J., Lanman, J. A., and Whitehouse, H. (2016). Collective synchrony increases prosociality towards non-performers and outgroup members. Br. J. Soc. Psychol. 55, 722-738. doi: 10.1111/bjso.12165

Rennung, M., and Göritz, A. S. (2016). Prosocial consequences of interpersonal synchrony: A meta-analysis. Z. Psychol. 224, 168-189. doi: 10.1027/2151-2604/ a000252

Rizzolatti, G., Cattaneo, L., Fabbri-Destro, M., and Rozzi, S. (2014). Cortical mechanisms underlying the organization of goal-directed actions and mirror neuron-based action understanding. Physiol. Rev. 94, 655-706. doi: 10.1152/ physrev.00009.2013

Rodriguez, V. (2013). The human nervous system: A framework for teaching and the teaching brain. Mind Brain Educ. 7, 2-12. doi: 10.1111/mbe.12000

Ryals, A. J., Rogers, L. M., Gross, E. Z., Polnaszek, K. L., and Voss, J. L. (2016). Associative recognition memory awareness improved by theta-burst stimulation of Frontopolar cortex. Cereb. Cortex 26, 1200-1210. doi: 10.1093/cercor/bhu311

Søndenå, P., Dalusio-King, G., and Hebron, C. (2020). Conceptualisation of the therapeutic alliance in physiotherapy: is it adequate? Musculoskelet. Sci. Pract. 46:102131. doi: 10.1016/j.msksp.2020.102131

Sowden, S., Wright, G. R., Banissy, M. J., Catmur, C., and Bird, G. (2015). Transcranial current stimulation of the temporoparietal junction improves lie detection. Curr. Biol. 25, 2447-2451. doi: 10.1016/j.cub.2015.08.014

Strauss, S., Calero, C. I., and Sigman, M. (2014). Teaching, naturally. Trends Neurosci. and Educ. 3, 38-43. doi: 10.1016/j.tine.2014.05.001

Szymanski, C., Müller, V., Brick, T. R., Von Oertzen, T., and Lindenberger, U. (2017). Hyper-transcranial alternating current stimulation: experimental manipulation of inter-brain synchrony. Front. Hum. Neurosci. 11:539. doi: 10.3389/ fnhum.2017.00539

Takeuchi, N., and Izumi, S. (2012). Noninvasive brain stimulation for motor recovery after stroke: mechanisms and future views. Stroke Res. Treat. 2012:584727. doi: 10.1155/2012/584727

Takeuchi, N., Mori, T., Suzukamo, Y., and Izumi, S. I. (2017). Integration of teaching processes and learning assessment in the prefrontal cortex during a video game teaching-learning task. Front. Psychol. 7:2052. doi: 10.3389/ fpsyg.2016.02052

Takeuchi, N., Mori, T., Suzukamo, Y., and Izumi, S.-I. (2019). Activity of prefrontal cortex in teachers and students during teaching of an insight problem. Mind Brain Educ. 13, 167-175. doi: 10.1111/mbe.12207

Takeuchi, N., Terui, Y., and Izumi, S. I. (2021). Oscillatory entrainment of neural activity between inferior frontoparietal cortices alters imitation performance. Neuropsychologia 150:107702. doi: 10.1016/j.neuropsychologia.2020.107702

Vaccaro, A. G., and Fleming, S. M. (2018). Thinking about thinking: A coordinatebased meta-analysis of neuroimaging studies of metacognitive judgements. Brain Neurosci. Adv. 2:2398212818810591. doi: 10.1177/2398212818810591

Vanbellingen, T., Pastore-Wapp, M., Kubel, S., Nyffeler, T., Schupfer, A. C., Kiefer, C., et al. (2020). Interhemispheric facilitation of gesturing: A combined theta burst stimulation and diffusion tensor imaging study. Brain Stimul. 13, 457-463. doi: 10.1016/j.brs.2019.12.013

Vogeley, K. (2017). Two social brains: neural mechanisms of intersubjectivity. Philos. Trans. R. Soc. Lond. Ser. B Biol. Sci. 372:20160245. doi: 10.1098/ rstb.2016.0245

Vosskuhl, J., Struber, D., and Herrmann, C. S. (2018). Non-invasive brain stimulation: A paradigm shift in understanding brain oscillations. Front. Hum. Neurosci. 12:211. doi: 10.3389/fnhum.2018.00211

Wass, S. V., Whitehorn, M., Marriott Haresign, I., Phillips, E., and Leong, V. (2020). Interpersonal neural entrainment during early social interaction. Trends Cogn. Sci. 24, 329-342. doi: 10.1016/j.tics.2020.01.006

Weiss, P. H., Achilles, E. I., Moos, K., Hesse, M. D., Sparing, R., and Fink, G. R. (2013). Transcranial direct current stimulation (tDCS) of left parietal cortex facilitates gesture processing in healthy subjects. J. Neurosci. 33, 19205-19211. doi: 10.1523/JNEUROSCI.4714-12.2013

Yao, Y. W., Chopurian, V., Zhang, L., Lamm, C., and Heekeren, H. R. (2021). Effects of non-invasive brain stimulation on visual perspective taking: A metaanalytic study. NeuroImage 242:118462. doi: 10.1016/j.neuroimage.2021.118462

Yeo, Y. X., Pestell, C. F., Bucks, R. S., Allanson, F., and Weinborn, M. (2021). Metacognitive knowledge and functional outcomes in adults with acquired brain injury: A meta-analysis. Neuropsychol. Rehabil. 31, 453-478. doi: 10.1080/09602011.2019.1704421

Zee, M., Koomen, H. M., and Van Der Veen, I. (2013). Student-teacher relationship quality and academic adjustment in upper elementary school: the role of student personality. J. Sch. Psychol. 51, 517-533. doi: 10.1016/j. jsp.2013.05.003

Zheng, L., Chen, C., Liu, W., Long, Y., Zhao, H., Bai, X., et al. (2018). Enhancement of teaching outcome through neural prediction of the students' knowledge state. Hum. Brain Mapp. 39, 3046-3057. doi: 10.1002/hbm.24059

Zhou, J. (2012). The effects of reciprocal imitation on teacher-student relationships and student learning outcomes. Mind Brain Educ. 6, 66-73. doi: 10.1111/j. 1751-228X.2012.01140.x

Conflict of Interest: The author declares that the research was conducted in the absence of any commercial or financial relationships that could be construed as a potential conflict of interest.

Publisher's Note: All claims expressed in this article are solely those of the authors and do not necessarily represent those of their affiliated organizations, or those of the publisher, the editors and the reviewers. Any product that may be evaluated in this article, or claim that may be made by its manufacturer, is not guaranteed or endorsed by the publisher.

Copyright (c) 2022 Takeuchi. This is an open-access article distributed under the terms of the Creative Commons Attribution License (CC BY). The use, distribution or reproduction in other forums is permitted, provided the original author(s) and the copyright owner(s) are credited and that the original publication in this journal is cited, in accordance with accepted academic practice. No use, distribution or reproduction is permitted which does not comply with these terms. 